\title{
RECURSOS TRÓFICOS UTILIZADOS POR PLEBEIA INTERMEDIA (APIDAE, MELIPONINI) EN LA LOCALIDAD DE BARITÚ, SALTA, ARgENTINA. CARACTERIZACIÓN BOTÁNICA DE SUS MIELES
}

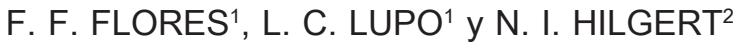

\begin{abstract}
Summary: Trophic resources used by Plebeia intermedia in the locality of Baritú, Salta, Argentina. Botanical characterization of its honeys. With the purpose of knowing the trophic resources used by Plebeia intermedia, a stingless bee of frequent use in Baritú locality (22 '28' 54.1" S; 64 45' 39.4" W; at 1546 masl), ten honey samples were melissopalynologically analyzed, collected in September and October of 2011 and in January of 2012. The qualitative examinations showed a certain specialization of these bees for using native and arboreal plants, and with flowers clustered in dense inflorescences (mass flowering). The dominant pollen types that originated monofloral honeys were Allophylus edulis (Sapindaceae), Blepharocalyx salicifolius (Myrtaceae) and Myrcianthes pseudomato (Myrtaceae), followed by Handroanthus ochraceus (Bignoniaceae), Ilex argentina (Aquifoliaceae), Parapiptadenia excelsa (Leguminosae) and Sambucus peruviana (Adoxaceae). Other pollen types observed in the samples, with frequency classes important minor or secondary, were Eupatorium (Compositae), Senecio (Compositae), Paullinia (Sapindaceae) and pollens of the families Boraginaceae and Rhamnaceae. The realized examinations in the honeys of Plebeia intermedia and results obtained in this study, constitute the first data on the trophic resources used by this species of stingless bee in the period spring - summer.
\end{abstract}

Key words: Melissopalynology, stingless bees, melliferous flora, Yungas.

\begin{abstract}
Resumen: Con la finalidad de conocer los recursos tróficos utilizados por Plebeia intermedia, una melipona de uso frecuente en la localidad de Baritú (22 $28^{\prime}$ 54,1" S; 64 45' 39,4" O; 1546 msnm), se efectuó el análisis polínico de diez muestras de miel colectadas en septiembre y octubre de 2011 y enero de 2012. Los exámenes cualitativos revelaron una cierta especialización de estas abejas por utilizar plantas nativas, de hábito arbóreo y con flores reunidas en inflorescencias abundantes (floraciones en masa). Los tipos polínicos dominantes presentes en las mieles monoflorales corresponden a las especies arbóreas Allophylus edulis (Sapindaceae), Blepharocalyx salicifolius (Myrtaceae) y Myrcianthes pseudomato (Myrtaceae), seguidos por Handroanthus ochraceus (Bignoniaceae), Ilex argentina (Aquifoliaceae), Parapiptadenia excelsa (Leguminosae) y Sambucus peruviana (Adoxaceae). Otros tipos polínicos observados en las muestras, con clases de frecuencia de menor importancia o secundario, fueron Eupatorium (Compositae), Senecio (Compositae), Paullinia (Sapindaceae) y pólenes de las familias Boraginaceae y Rhamnaceae. Los análisis efectuados en las mieles de Plebeia intermedia y los resultados obtenidos en este estudio, constituyen los primeros datos sobre los recursos tróficos utilizados por esta especie de melipona en el período primavera - verano.
\end{abstract}

Palabras clave: Melisopalinología, meliponas, flora melífera, Yungas.

\footnotetext{
${ }^{1}$ Centro de Investigación y Transferencia (CIT - Jujuy), CONICET. Laboratorio de Palinología. Facultad de Ciencias Agrarias, UNJu. Alberdi 47 C. P. 4600. San Salvador de Jujuy, Jujuy, Argentina. E-mail: fabiofflores@gmail.com

${ }^{2}$ Instituto de Biología Subtropical (IBS), UNAM- CONICET, Facultad de Ciencias Forestales, Centro de Investigaciones del Bosque Atlántico (CelBA), Bertoni 85. C. P. 3370. Puerto Iguazú, Misiones. E-mail: normahilgert@yahoo.com.ar
} 


\section{INTRODUCCIÓN}

Las meliponas o abejas sin aguijón son insectos eusociales que viven en colonias permanentes, encontradas en regiones tropicales y subtropicales del mundo (Aguilera Peralta \& Ferrufino, 2004). En la región Neotropical, su distribución se extiende hasta los $35^{\circ}$ latitud Sur (Michener, 2007) y en Argentina se las encuentra en las provincias fitogeográficas Selva Paranaense, el Chaco y las Yungas (Roig Alsina et al., 2013). Pertenecen a la familia Apidae, tribu Meliponini Lepeletier (Camargo \& Pedro, 2013).

Los antecedentes sobre los recursos tróficos usados por estos insectos melíferos tienen distinto grado de desarrollo, según el país y las regiones. Esta información permite identificar las especies de plantas que aportan néctar y/o polen, resultando listados florísticos, como los confeccionados por Martínez Hernández et al. (1993), Knoll et al. (1994), Maia Silva et al. (2012) y Da Silva (2014). Esta información orienta al productor acerca del manejo de las colmenas; aporta elementos explicativos a la hora de evaluar características físico-químicas, microbiológicas y antioxidantes de las mieles, así como también contribuye a comprender la relación existente entre las plantas, las colmenas de meliponas y el uso diferencial otorgado a estas mieles por los grupos humanos.

Estudios de esta índole presentan avances importantes en países donde se emplean sus productos, tomados de nidos silvestres $\mathrm{y} / \mathrm{o}$ de colmenas criadas de modo racional. Por ejemplo, en Brasil se llevaron a cabo estudios sobre los recursos tróficos utilizados por las meliponas, a través del registro de las visitas florales (Ramalho, 2004; Monteiro \& Ramalho, 2010) y del análisis de las mieles o reservas de polen (Iwama \& Melhem, 1979; Ramalho et al., 1985; Conceição, 2013); como así también, se estudiaron los aspectos competitivos y el solapamiento de nichos tróficos frente a abejas exóticas (Cortopassi Laurino \& Ramalho, 1988; Menezes Pedro \& Camargo, 1991; Hilgert Moreira et al., 2013). Estudios de esta índole se encuentran compendiados en el trabajo de Ramalho et al. (1990).

En Argentina, los avances actuales incluyen estudios de los sitios de nidificación (Colleselli \& Gómez de Olivera, 2008; Vossler, 2012; Sparagnino et al., 2014), así como el uso de los recursos tróficos.
En este último aspecto, en la provincia fitogeográfica Chaqueña (Cabrera, 1971), pueden citarse los trabajos sobre visitas florales de Geotrigona argentina Camargo \& Moure, Lestrimelitta chacoana Roig Alsina, Melipona orbignyi (Guérin), Plebeia catamarcensis (Holmberg), P. molesta (Puls), Tetragonisca fiebrigi Schwarz y Scaptotrigona jujuyensis Schrottky (Vossler, 2012), como también el examen polínico de las mieles, reservas de polen y cargas corbiculares de G. argentina, S. jujuyensis y T. fiebrigi (Vossler et al., 2010, 2014; Basilio et al., 2013; Vossler, 2015). En la provincia fitogeográfica de las Yungas, las investigaciones sobre esta temática son aún incipientes, encontrándose como único registro el examen de las mieles de $T$. angustula (Flores \& Sánchez, 2010).

En este contexto y con el objetivo de conocer las principales fuentes de néctar empleadas por Plebeia intermedia Wille (Apidae, Meliponini) (Camargo \& Pedro, 2013) en la localidad de Baritú, se presentan los resultados de los análisis palinológicos efectuados en muestras de mieles.

\section{Materiales y Métodos}

El área de estudio corresponde a la localidad de Baritú, perteneciente al departamento de Santa Victoria de la provincia de Salta $\left(22^{\circ} 28^{\prime} 54,1^{\prime \prime} \mathrm{S}\right.$ $64^{\circ} 45^{\prime}$ 39,4" O; $\left.1546 \mathrm{msnm}\right)$. Este paraje, desde el punto de vista fitogeográfico, está ubicado en una transición entre los distritos de Selvas Montanas y Bosques Montanos de Yungas (Cabrera, 1971; Ramadori, 1995) y colinda con el Parque Nacional Baritú (Figura 1).

En el marco de un estudio etnobiológico sobre el uso de mieles silvestres, se mantuvieron entrevistas con los pobladores locales, observándose que Plebeia intermedia (llamada "mansita"), es la especie de melipona utilizada con mayor frecuencia y con usos más diversos. La especie tiene una distribución en la región Neotropical (hasta ahora sólo había sido citada en Cochabamba y La Paz, en Bolivia) (Camargo \& Pedro, 2013), siendo éste el primer registro para Argentina; lo que demuestra una distribución más amplia y austral. Las mieles con las que se trabajó fueron suministradas por los informantes. Se analizaron diez mieles diferentes, 3 colectadas en septiembre y 5 en octubre del 2011 y 2 en enero de 2012. 


\section{F. F. Flores et al. - Caracterización botánica de mieles de Plebeia intermedia de Baritú}

De cada miel se analizó una muestra, las que fueron procesadas según Louveaux et al. (1978) y posteriormente acetolizadas (Erdtman, 1960). Se contaron 1000 granos de polen como mínimo por preparado, y se establecieron las clases de frecuencia de acuerdo a Louveaux et al. (1978). A su vez, según Feller Demalsy et al. (1987), se estimó la frecuencia de ocurrencia de los tipos polínicos en el total de las muestras como Muy Frecuentes (presentes en más del 50\%), Frecuentes (20-50\%), Poco Frecuentes $(10-20 \%)$ y Raros $(<10 \%)$. De modo complementario, se registraron separadamente los tipos polínicos procedentes de plantas con polinización anemófila o sin néctar, debido a que no contribuyen en la elaboración de las mieles y su presencia en las mismas puede distorsionar la caracterización botánica. Los datos registrados fueron excluidos del número total de granos de polen, antes del cálculo de las clases de frecuencias (Louveaux et al., 1978).
Los tipos polínicos se identificaron a nivel especie, género y familia botánica. Para esto se consultaron atlas palinológicos (Heusser, 1971; Markgraf \& D'Antoni, 1978; Pire et al., 1998, 2001, 2006) y la palinoteca de referencia del área de estudio y del Laboratorio de Palinología (PALJUA). En aquellos casos en los que no fue posible asignar una categoría, se utilizó el término "tipo", con lo cual se presume que el polen tiene semejanza con alguna especie, género o familia y cuyos datos deben ser corroborados en estudios posteriores. Para la nomenclatura de los tipos polínicos se siguieron las indicaciones de Joosten \& De Klerk (2002) y De Klerk \& Joosten (2007).

Las mieles fueron consideradas monoflorales cuando presentaban algún tipo polínico dominante (mayor al $45 \%$ del polen total, considerando las excepciones planteadas por Louveaux et al., 1978). Aquéllas que no mostraban un polen dominante se
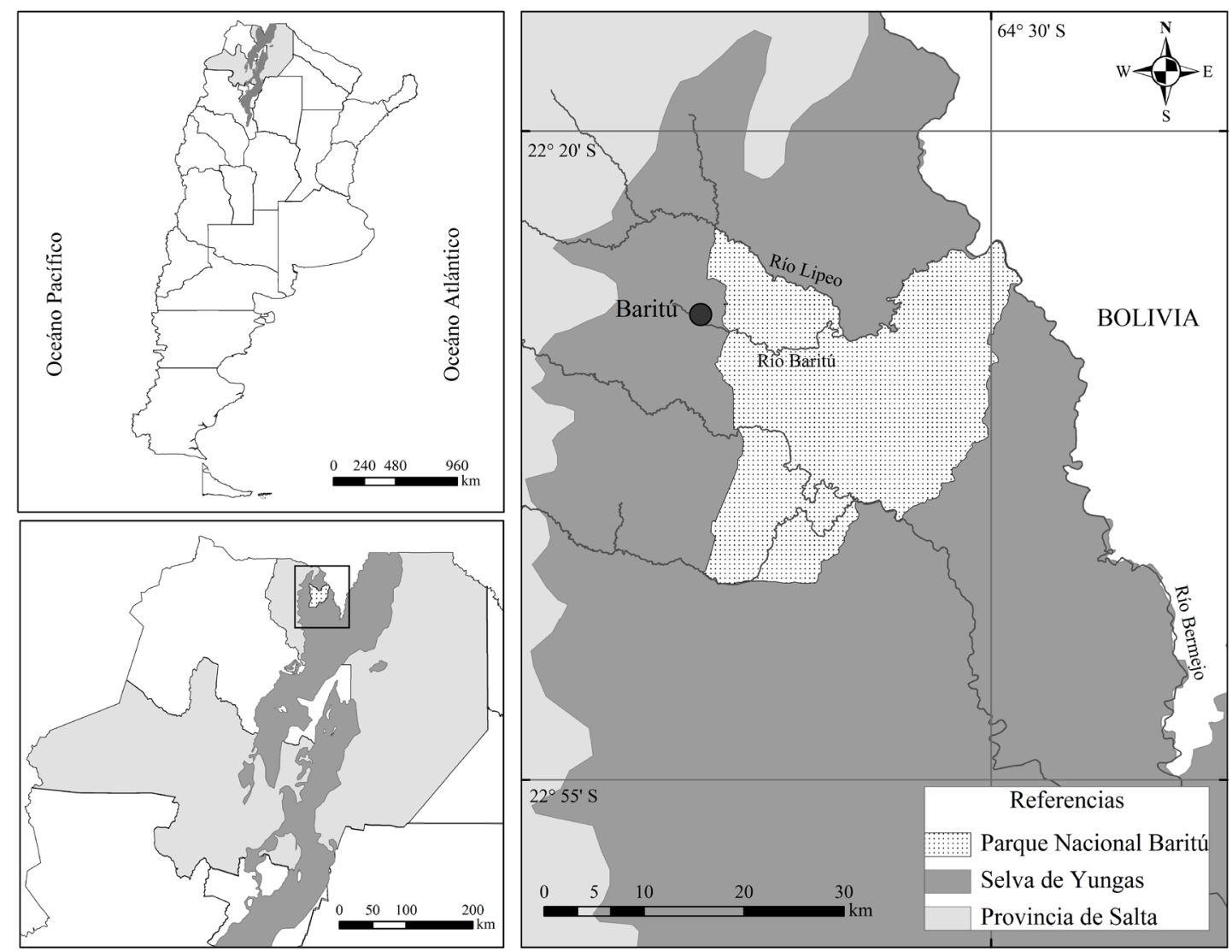

Fig. 1. Área de estudio ubicada colindante al Parque Nacional Baritú y emplazada en la provincia fitogeográfica de las Yungas. 
clasificaron como multiflorales. Las observaciones se realizaron con microscopio óptico Zeiss ICS KF2 y las microfotografías se tomaron con una cámara digital Leica ICC50 combinado al microscopio Leica DM 500. Los preparados obtenidos forman parte de la palinoteca de referencia PAL-JUA del Laboratorio de Palinología - Facultad de Ciencias Agrarias - Universidad Nacional de Jujuy.

Con el fin de ilustrar el total de tipos polínicos hallados en las mieles se efectuó un diagrama polínico empleando el programa Tilia 1.7.16 (Grimm, 2011) ordenando las muestras de acuerdo a las fechas de colecta. Para la confección de la base de datos, se ingresaron las 10 muestras con una cantidad de 55 variables, equivalentes a los tipos polínicos identificados. Además, se realizó un agrupamiento de las muestras de miel, mediante un análisis de cluster Coniss (Constrained Incremental Sum of Squares) con base en la frecuencia, variabilidad y abundancia de los tipos polínicos. Para la comparación de las muestras, el examen empleó el Coeficiente de Disimilitud de Edwards \& Cavalli-Sforza.

Complementariamente, con la finalidad de evaluar el aporte de las plantas según sus formas de vida se realizaron figuras asignando a cada tipo polínico la categoría del hábito de la especie vegetal de acuerdo a Zuloaga et al. (2008) y calculando la frecuencia porcentual (porcentaje de tipos polínicos por hábito) y la abundancia porcentual (porcentaje de individuos por tipo polínico por hábito). Para la designación del hábito de los tipos polínicos identificados a nivel de género botánico, en cada caso se priorizó el hábito de las especies vegetales presentes en el área de estudio. En aquellos casos en los que no fue posible definir al tipo polínico, se agruparon en la categoría de Hábito indefinido. Para la nomenclatura botánica se siguió el criterio de The Plants List (www.theplantlist.org).

\section{Resultados}

\section{Análisis melisopalinológico}

Durante la caracterización botánica de las mieles, en las muestras se observaron 54 tipos polínicos, identificados al menor nivel de determinación taxonómico posible: 14 a nivel de especie, 27 a nivel de género y 13 a nivel de familia botánica. A su vez, se hallaron 3 tipos polínicos con porcentajes inferiores al $1 \%$ que fueron agrupados en la categoría indeterminados. Los resultados del análisis cualitativo se presentan en la Tabla 1, en las que se observan los tipos polínicos encontrados con sus respectivas clases de frecuencia y, asimismo, la frecuencia de ocurrencia de cada uno en el total de las muestras.

La riqueza polínica por miel varió de 10 a 24 tipos polínicos, con un promedio de 18,9. En los pólenes identificados se encuentran representadas 33 familias botánicas, siendo la de mayor riqueza la familia Compositae con 5 tipos, seguidas por Euphorbiaceae y Sapindaceae con 4 tipos; Leguminosae con 3 tipos; Amaranthaceae, Bignoniaceae, Cactaceae, Lamiaceae, Myrtaceae, Rosaceae, Rutaceae, Solanaceae y Verbenaceae con 2 tipos cada una, y las demás familias representadas por uno solo.

De acuerdo a las clases de frecuencia (Louveaux et al., 1978), se encontró que el 3,2\% son tipos polínicos Dominantes (D: $>45 \%$ ), el 6,3\% Secundarios (S: 15 45\%), un 11,1\% de Menor importancia (M: 3-15\%) y el 79,4\% en Trazas (T: <3\%) (Fig. 2).

Del examen efectuado, el $40 \%$ de las muestras se clasificaron como mieles multiflorales y el 60\% mieles monoflorales de Allophylus edulis (Sapindaceae) (20\%), Blepharocalyx salicifolius (Myrtaceae) (20\%) y Myrcianthes pseudomato (Myrtaceae) (20\%); Fig. 3, 4 y 5.

Conforme a su origen, pudo verse que el $96 \%$ de los tipos polínicos proceden de especies vegetales propias de las Yungas, el 2\% de plantas exóticas y/o cultivadas (e. g. Citrus, Rutaceae) y un $2 \%$ a pólenes a los que no se les pudo asignar una filiación taxonómica (Fig. 6).

Entre los pólenes provenientes de plantas con polinización anemófila o sin néctar, en las muestras se hallaron tipos polínicos de Alnus acuminata (Betulaceae), Celtis (Cannabaceae), Juglans australis (Juglandaceae), tipo Rumex (Polygonaceae) y Zea mays (Poaceae) (Tabla 1, Fig.7).

Frecuencia de ocurrencia de los tipos polínicos en el total de las muestras de miel de Plebeia intermedia

Al analizar la frecuencia de ocurrencia de los tipos polínicos en el total de las muestras se encontraron 11 pólenes Muy Frecuentes ( $>50 \%$ de las muestras): Allophylus edulis, Eupatorium (Compositae), Handroanthus ochraceus (Bignoniaceae), Heteropterys (Malpighiaceae), Ilex 
F. F. Flores et al. - Caracterización botánica de mieles de Plebeia intermedia de Baritú

Tabla 1. Tipos polínicos identificados en las mieles de Plebeia intermedia de la localidad de Baritú.

Representación de las clases de frecuencia D: polen dominante (>45\%); S: polen secundario (15-45\%);

M: polen de menor importancia (3-15\%); T: polen en traza $(<3 \%)$. FO: Frecuencia de ocurrencia de los tipos polínicos en el total de las muestras. * Plantas de origen exótico y/o cultivado. + Presencia en la muestra del polen de plantas con polinización anemófila o sin néctar.

\begin{tabular}{|c|c|c|c|c|c|c|c|c|c|c|c|}
\hline \multirow{2}{*}{ Tipos polínicos } & 1 & 2 & 3 & 4 & 5 & 6 & 7 & 8 & 9 & 10 & \multirow{2}{*}{ FO (\%) } \\
\hline & Set1 & Set2 & Set3 & Oct1 & Oct2 & Oct3 & Oct4 & Oct5 & Ene1 & Ene2 & \\
\hline \multicolumn{12}{|l|}{ Acanthaceae } \\
\hline Justicia & & & $\mathrm{T}$ & & & & & & $\mathrm{T}$ & & 20 \\
\hline \multicolumn{12}{|l|}{ Adoxaceae } \\
\hline Sambucus peruviana & & & & & & & & & M & $M$ & 20 \\
\hline \multicolumn{12}{|l|}{ Amaranthaceae } \\
\hline Alternanthera & & & $T$ & & & & $\mathrm{~T}$ & & & & 20 \\
\hline Chamissoa & & & $T$ & $\mathrm{~T}$ & & & & & & & 20 \\
\hline \multicolumn{12}{|l|}{ Anacardiaceae } \\
\hline Schinus & & $\mathrm{T}$ & & & & & & & & & 10 \\
\hline Apiaceae & & $\mathrm{T}$ & & & & & & $\mathrm{T}$ & $\mathrm{T}$ & $\mathrm{T}$ & 40 \\
\hline \multicolumn{12}{|l|}{ Aquifoliaceae } \\
\hline Ilex argentina & $\mathrm{T}$ & $\mathrm{T}$ & $T$ & $\mathrm{~T}$ & $\mathrm{~T}$ & $M$ & $\mathrm{~T}$ & $\mathrm{~S}$ & $M$ & $M$ & 100 \\
\hline \multicolumn{12}{|l|}{ Bignoniaceae } \\
\hline Dolichandra unguis-cati & & & $T$ & $\mathrm{~T}$ & $\mathrm{~T}$ & & & & & & 30 \\
\hline Handroanthus ochraceus & M & $\mathrm{T}$ & M & $\mathrm{T}$ & $T$ & & $\mathrm{~T}$ & & & $\mathrm{~T}$ & 70 \\
\hline Boraginaceae & $\mathrm{T}$ & $\mathrm{T}$ & & $\mathrm{T}$ & $\mathrm{S}$ & & & & $\mathrm{T}$ & $\mathrm{T}$ & 60 \\
\hline Brassicaceae & & & & & $\mathrm{T}$ & & $\mathrm{T}$ & $\mathrm{T}$ & & & 30 \\
\hline Cactaceae & & & & & $\mathrm{T}$ & & $\mathrm{T}$ & $\mathrm{T}$ & & & 30 \\
\hline Pereskia & & & $T$ & & & & & & $\mathrm{~T}$ & & 20 \\
\hline Compositae & $\mathrm{T}$ & & & & & & $\mathrm{T}$ & $\mathrm{T}$ & & $\mathrm{T}$ & 40 \\
\hline Eupatorium & $\mathrm{T}$ & & $\mathrm{S}$ & M & $T$ & $\mathrm{~T}$ & $\mathrm{~T}$ & M & $\mathrm{T}$ & $\mathrm{T}$ & 90 \\
\hline Senecio & & $\mathrm{T}$ & $M$ & $\mathrm{~T}$ & $\mathrm{~T}$ & & & & & & 40 \\
\hline Tipo Gnaphalium & & & $\mathrm{T}$ & & & & & & & & 10 \\
\hline Vernonia & & & $\mathrm{T}$ & & & & & & & & 10 \\
\hline \multicolumn{12}{|l|}{ Cucurbitaceae } \\
\hline Sicyos & $\mathrm{T}$ & $\mathrm{T}$ & $\mathrm{T}$ & & $\mathrm{T}$ & & & $\mathrm{T}$ & & & 50 \\
\hline \multicolumn{12}{|l|}{ Eleocarpaceae } \\
\hline Crinodendron tucumanum & & & & & & & & $\mathrm{T}$ & & & 10 \\
\hline Euphorbiaceae & $\mathrm{T}$ & $\mathrm{T}$ & & $\mathrm{T}$ & & & & $\mathrm{T}$ & & & 40 \\
\hline Croton & $\mathrm{T}$ & & & & $\mathrm{T}$ & & & $\mathrm{T}$ & $\mathrm{T}$ & $\mathrm{T}$ & 50 \\
\hline Sapium & & & & & & $T$ & & & & & 10 \\
\hline Sebastiana & & & & & & & & & $\mathrm{T}$ & $\mathrm{T}$ & 20 \\
\hline \multicolumn{12}{|l|}{ Flacourtiaceae } \\
\hline tipo Casearia & & & $\mathrm{T}$ & & & & & & & & 10 \\
\hline Lamiaceae & & & & & & & $\mathrm{T}$ & & & & 10 \\
\hline Hyptis & $\mathrm{T}$ & & & $\mathrm{T}$ & & & & & & & 20 \\
\hline \multicolumn{12}{|l|}{ Leguminosae } \\
\hline Acacia & & & & & & $\mathrm{T}$ & & & & & 10 \\
\hline Anadenanthera colubrina & & & & & & & & & $\mathrm{T}$ & & 10 \\
\hline Parapiptadenia excelsa & $\mathrm{T}$ & $\mathrm{T}$ & & $\mathrm{T}$ & $\mathrm{T}$ & $\mathrm{T}$ & M & M & $\mathrm{S}$ & M & 90 \\
\hline Loranthaceae & & & & & $\mathrm{T}$ & & & & & & 10 \\
\hline Malpighiaceae & & & & & & & & & & & \\
\hline
\end{tabular}


Bol. Soc. Argent. Bot. 50 (4) 2015

\begin{tabular}{|c|c|c|c|c|c|c|c|c|c|c|c|}
\hline \multirow{2}{*}{ Tipos polínicos } & 1 & 2 & 3 & 4 & 5 & 6 & 7 & 8 & 9 & 10 & \multirow{2}{*}{$\mathrm{FO}(\%)$} \\
\hline & Set1 & Set2 & Set3 & Oct1 & Oct2 & Oct3 & Oct4 & Oct5 & Ene1 & Ene2 & \\
\hline Heteropterys & & & & $\mathrm{T}$ & $\mathrm{T}$ & & $\mathrm{T}$ & $\mathrm{T}$ & $\mathrm{T}$ & $\mathrm{T}$ & 60 \\
\hline Meliaceae & & $\mathrm{T}$ & & & & & & & & & 10 \\
\hline Moraceae & & & & $T$ & $\mathrm{~T}$ & & & & & & 20 \\
\hline \multicolumn{12}{|l|}{ Myrtaceae } \\
\hline Blepharocalyx salicifolius & $\mathrm{D}$ & D & M & & & S & & $S$ & & & 50 \\
\hline Myrcianthes pseudomato & & & & M & $\mathrm{T}$ & D & D & $\mathrm{s}$ & $\mathrm{S}$ & $\mathrm{s}$ & 70 \\
\hline \multicolumn{12}{|l|}{ Primulaceae } \\
\hline Myrsine coriacea & $T$ & $\mathrm{~T}$ & $T$ & & $\mathrm{~T}$ & & & $\mathrm{~T}$ & $\mathrm{~T}$ & $T$ & 70 \\
\hline Rhamnaceae & $\mathrm{T}$ & & & $\mathrm{M}$ & $T$ & & & $T$ & $\mathrm{~T}$ & $T$ & 60 \\
\hline \multicolumn{12}{|l|}{ Rosaceae } \\
\hline Prunus tucumanensis & & $T$ & & & & & & & & & 10 \\
\hline Tipo Acaena & & & & & & & & & & $\mathrm{T}$ & 10 \\
\hline \multicolumn{12}{|l|}{ Rubiaceae } \\
\hline Randia micracantha & & & $T$ & $T$ & $T$ & & $T$ & & & $T$ & 50 \\
\hline \multicolumn{12}{|l|}{ Rutaceae } \\
\hline Citrus * & $\mathrm{T}$ & & & & & & & & & $\mathrm{T}$ & 20 \\
\hline Zanthoxylum & & & $\mathrm{T}$ & $\mathrm{T}$ & & $\mathrm{T}$ & & & & & 30 \\
\hline \multicolumn{12}{|l|}{ Sapindaceae } \\
\hline Allophylus edulis & $\mathrm{T}$ & $\mathrm{M}$ & $\mathrm{s}$ & D & D & $\mathrm{T}$ & $\mathrm{M}$ & $\mathrm{M}$ & $\mathrm{s}$ & $\mathrm{s}$ & 100 \\
\hline Matayba & & $\mathrm{T}$ & $T$ & $\mathrm{~T}$ & & $T$ & & & & $T$ & 50 \\
\hline Paullinia & & & M & & & & & & M & $\mathrm{T}$ & 30 \\
\hline Serjania & & $\mathrm{T}$ & $\mathrm{T}$ & & $\mathrm{T}$ & & & & & & 30 \\
\hline Solanaceae & & & $T$ & & & & & & & & 10 \\
\hline Solanum & $T$ & & & & & & $T$ & & $\mathrm{~T}$ & & 30 \\
\hline \multicolumn{12}{|l|}{ Tropaeolaceae } \\
\hline Tropaeolum & & $T$ & $T$ & & $\mathrm{~T}$ & & & & & & 30 \\
\hline \multicolumn{12}{|l|}{ Ulmaceae } \\
\hline Phyllostylon rhamnoides & & $\mathrm{T}$ & $T$ & $\mathrm{~T}$ & & & $\mathrm{~T}$ & & $\mathrm{~T}$ & $T$ & 60 \\
\hline Verbenaceae & & & & & & & & & & $T$ & 10 \\
\hline Tipo Aloysia & & & & & & & & & $T$ & $T$ & 20 \\
\hline \multicolumn{12}{|l|}{ Vitaceae } \\
\hline Cissus & $\mathrm{T}$ & & & & & & & & $T$ & $T$ & 30 \\
\hline Indeterminados & $T$ & $T$ & & $T$ & & & & & & & 30 \\
\hline Riqueza polínica & 18 & 19 & 24 & 20 & 21 & 10 & 15 & 17 & 21 & 24 & \\
\hline
\end{tabular}

Tipos polínicos de plantas con polinización anemófila o sin néctar

\begin{tabular}{|l|c|c|c|c|c|c|c|c|c|c|}
\hline Betulaceae & & & & & & & & & & \\
\hline$\quad$ Alnus acuminata & & + & + & & & + & & & & \\
\hline Cannabaceae & & & & & & & & & & \\
\hline$\quad$ Celtis & + & + & + & + & + & + & + & + & + & + \\
\hline Juglandaceae & & & & & & & & & & \\
\hline$\quad$ Juglans australis & & & & & & + & + & + & & \\
\hline Poaceae & & & & & & & & & & \\
\hline Zea mays * & + & & & & & & & & & \\
\hline Polygonaceae & & & & & & & & & & \\
\hline$\quad$ Tipo Rumex & & & + & & & & + & + & + & + \\
\hline
\end{tabular}




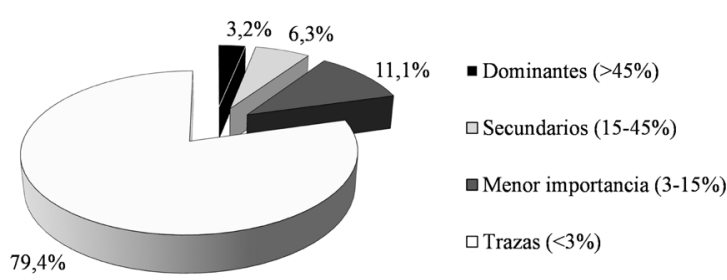

Fig. 2. Representatividad de los tipos polínicos de acuerdo a las clases de frecuencia.

argentina (Aquifoliaceae), Myrcianthes pseudomato, Myrsine coriacea (Primulaceae), Parapiptadenia excelsa (Leguminosae), Phyllostylon rhamnoides (Ulmaceae) y tipos polínicos de las familias Boraginaceae y Rhamnaceae; 28 Frecuentes (20$50 \%$ de las muestras): Blepharocalyx salicifolius, Citrus, Dolichandra unguis-cati (Bignoniaceae), Matayba (Sapindaceae), Paullinia (Sapindaceae), Randia micracantha (Rubiaceae), Sambucus peruviana (Adoxaceae), Senecio (Compositae), Serjania (Sapindaceae), Sicyos (Cucurbitaceae), pólenes de Euphorbiaceae, Moraceae, entre otros; 15 Poco Frecuentes (10-20\% de las muestras): Acacia (Leguminosae), Crinodendron tucumanum (Elaeocarpaceae), Prunus tucumanensis (Rosaceae), Schinus (Anacardiaceae) y pólenes de Lamiaceae, Meliaceae, Solanaceae, entre otros; y ninguno en la categoría Raros $(<10 \%)$, Tabla 1.

\section{Diagrama polínico}

Al evaluar el diagrama polínico elaborado y el análisis cluster obtenido (Fig. 8), se observan los tipos polínicos ordenados en dos grupos principales (denominados en adelante A y B). El grupo A se destaca por la asociación de Allophylus edulis y Blepharocalyx salicifolius con porcentajes que varían entre $4 \%$ a $77 \%$ y $13 \%$ a $88 \%$ respectivamente; ambos son los tipos polínicos dominantes que dieron origen a mieles monoflorales. En segunda instancia se encuentran acompañados por la presencia de Cissus (Vitaceae) (1,5\%), Eupatorium (1,6 a 20\%), Handroanthus ochraceus (5 a 6\%), Hyptis (Lamiaceae) (1,5\%), Ilex argentina (1,9\% a 2,2\%), Myrsine coriacea (1,6\%), Myrcianthes pseudomato (3\%), Paullinia (3\%), Senecio (11\%) y pólenes de las familias Boraginaceae (18\%) y Rhamnaceae (8\%).

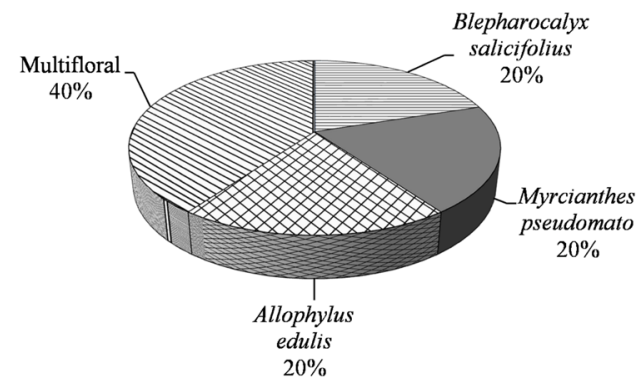

Fig. 3. Clasificación de las mieles de Plebeia intermedia de la localidad de Baritú.

El grupo B está representado por la asociación de pólenes de Allophylus edulis (valores de 0,5 a 26\%), Blepharocalyx salicifolius (27\% a 35\%) y Myrcianthes pseudomato (valores de $28 \%$ a $87 \%$ ) que permitieron definir mieles monoflorales. Complementariamente, se encuentran acompañados por la presencia de Eupatorium (7\%), Ilex argentina (valores de 2 a 22\%), Parapiptadenia excelsa (3 a 17\%), Paullinia (4\%) y se recalca en las muestras de enero la presencia de Sambucus peruviana con valores de 5\% a $12 \%$.

El aporte nectarífero y las formas de vida de las plantas

Al evaluar el hábito de la especie vegetal y la frecuencia porcentual de los tipos polínicos de acuerdo a las categorías propuestas por Zuloaga et al. (2008) se pudo ver que el 35\% corresponden a especies vegetales arbóreas, un $18 \%$ a especies arbustivas, el $11 \%$ a hierbas, un $5 \%$ a lianas, el $4 \%$ a enredaderas y un $2 \%$ a especies de hábito parásito. Respecto a los tipos polínicos de hábito indefinido se observó un valor de $21 \%$ y el $5 \%$ corresponden a pólenes no determinados (Fig. 9A). Asimismo, al evaluar el hábito de la planta y la abundancia porcentual de los tipos polínicos, se mantiene el predominio de los árboles (Fig. 9B).

\section{Discusión y Conclusiones}

En las muestras analizadas se hallaron 54 tipos polínicos, de los cuales unos pocos se categorizaron como de uso frecuente. Esta tendencia también fue observada en la región Chaqueña argentina, donde las colmenas de Geotrigona argentina visitaron una 
Bol. Soc. Argent. Bot. 50 (4) 2015

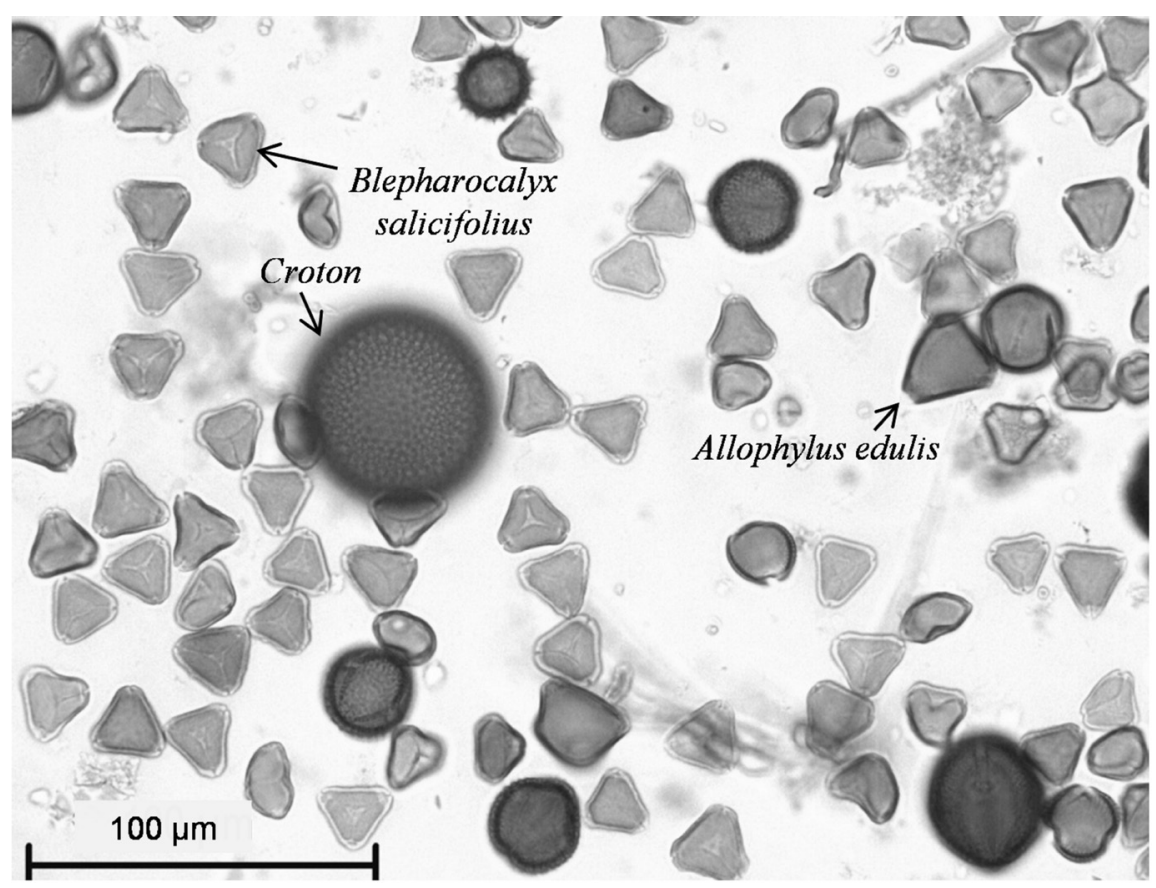

Fig. 4. Muestra monofloral de Blepharocalyx salicifolius en mieles de Plebeia intermedia de la localidad de Baritú.

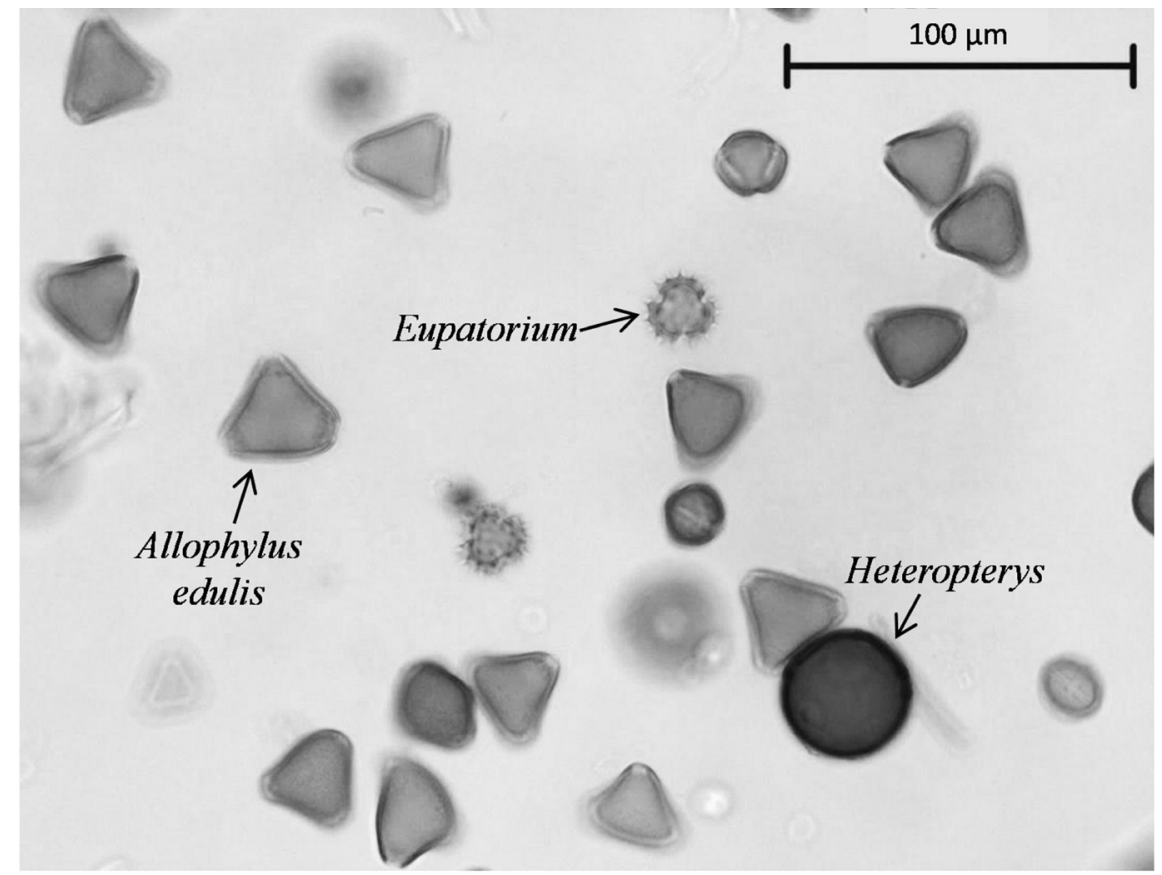

Fig. 5. Muestra monofloral de Allophylus edulis en mieles de Plebeia intermedia de la localidad de Baritú. 


\section{F. F. Flores et al. - Caracterización botánica de mieles de Plebeia intermedia de Baritú}

amplia variedad de plantas, pero con una constancia de uso concentrada en un grupo pequeño de árboles y arbustos leñosos (Vossler et al., 2010).

La familia Compositae fue la más representada, con 5 tipos polínicos, sin embargo, por la frecuencia de uso, se destacaron los representantes de la familia Myrtaceae, seguido de las familias Sapindaceae, Aquifoliacae y Leguminosae. La utilización de recursos tróficos provenientes de la familia Myrtaceae, coinciden con lo observado en sectores de Brasil, donde fueron aprovechados por colmenas de Plebeia remota (Ramalho et al., 1985), Tetragonisca angustula angustula (Iwama \& Melhem, 1979) y otras especies de meliponas (Ramalho et al., 1990); como así también, en sectores de las Yungas argentinas, donde la familia fue de importancia nectarífera para las colmenas de T. angustula (Flores \& Sánchez, 2010). El gran número de especies vegetales que presenta la familia en las zonas neotropicales, proporciona un conjunto mayor de recursos tróficos disponibles para las abejas (Ramalho et al., 1990), lo cual

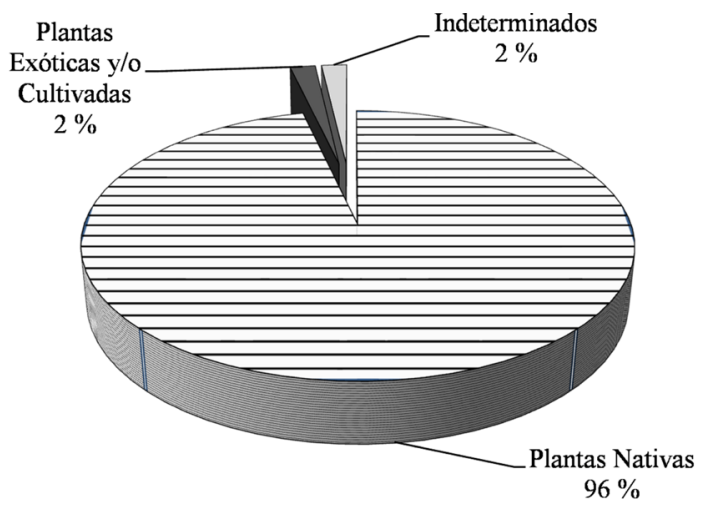

Fig. 6. Representatividad de las plantas nativas y exóticas/cultivadas en las mieles de Plebeia intermedia.

probablemente explique la preferencia de uso de estos vegetales por parte de las abejas.

Las especies Allophylus edulis, Blepharocalyx salicifolius y Myrcianthes pseudomato, las más frecuentemente observadas en las muestras estudiadas
A

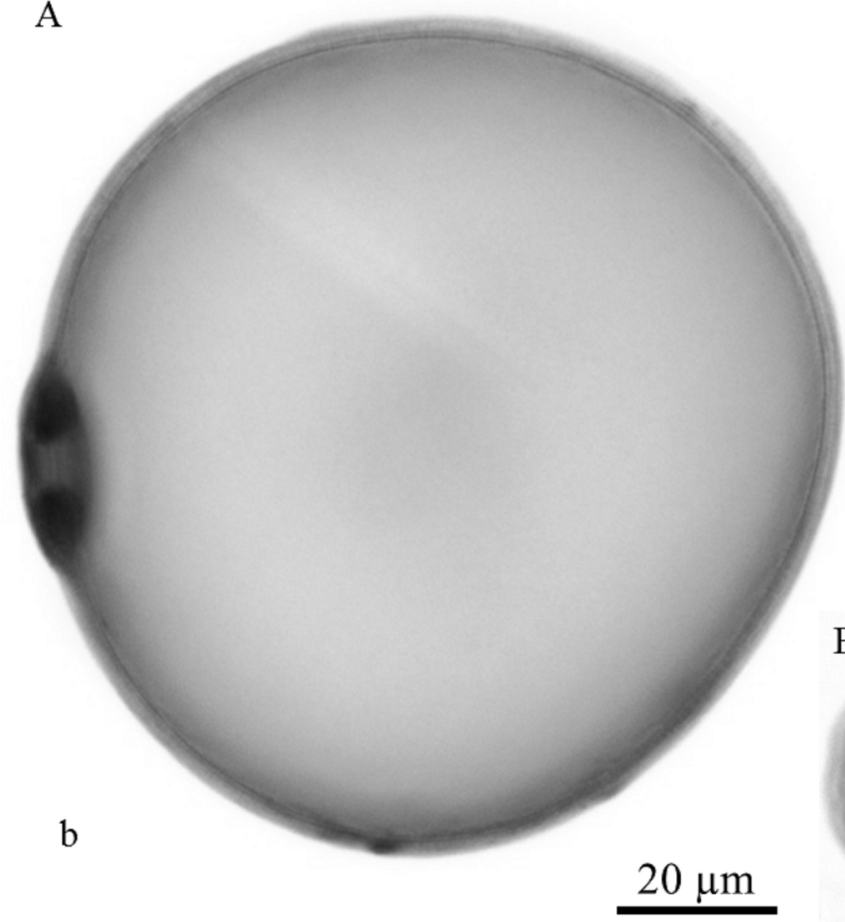

B

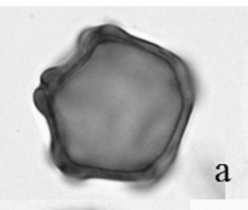

C
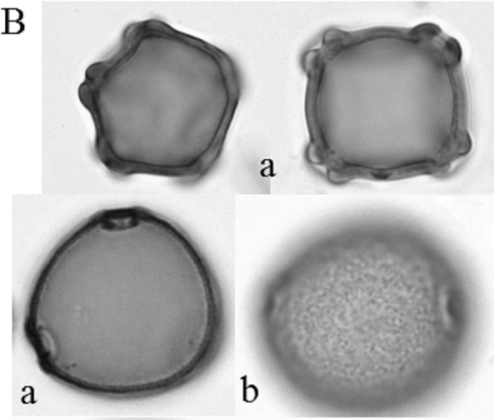

$\mathrm{D}$

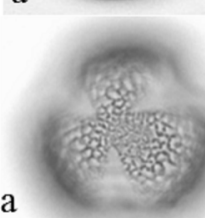

b
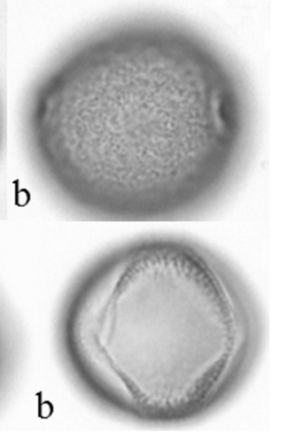

E

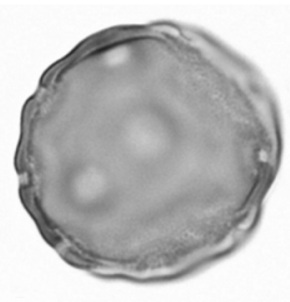

Fig. 7. Tipos polínicos de plantas con polinización anemófila o sin néctar. A: Zea mays. B: Alnus acuminata. C: Celtis. D: Tipo Rumex. E: Juglans australis. a: Vista polar. b: Vista ecuatorial. 
Bol. Soc. Argent. Bot. 50 (4) 2015

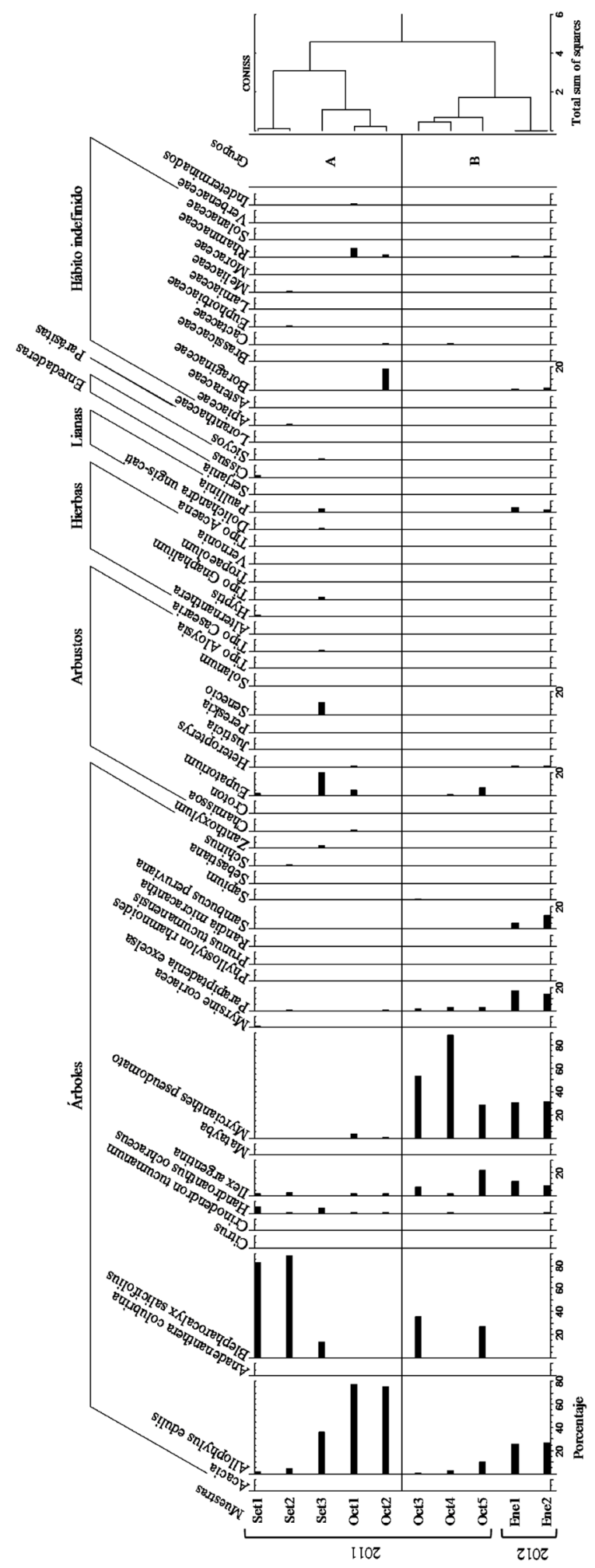

Fig. 8. Diagrama polínico de muestras de miel de Plebeia intermedia de la localidad de Baritú. 
y con las que se definieron mieles monoflorales, fueron halladas con roles tróficos semejantes en mieles de Apis mellifera procedentes de ambientes yungueños de la provincia de Jujuy (Sánchez, 2013; Méndez, 2014), lo cual recalca la importancia trófica que tienen para las abejas melíferas. De estas tres especies arbóreas presentes en los distritos Selva Montana y Bosque Montano de las Yungas (Morales et al., 1995; Morales \& Brown, 1998; Brown, 2007; Brown \& Malizia, 2007), Myrcianthes pseudomato prospera exclusivamente en las provincias de Tucumán, Jujuy y Salta, en Argentina (http://www.darwin.edu.ar/ Proyectos/FloraArgentina/DetalleEspecie.asp?form $\mathrm{a}=\&$ variedad $=\&$ subespecie $=\&$ especie $=$ pseudomat o\&genero=Myrcianthes\&espcod=144); las tres son especies tolerantes a las sombras que caracterizan tipos estructurales pertenecientes a bosques maduros (Pinazo et al., 2003) y con recompensas florales (néctar y polen) disponibles durante la época primaveral. En efecto, Allophylus edulis presenta individuos de 4 a 8 $\mathrm{m}$ de altura, con inflorescencias aprovechables durante el período de agosto a noviembre; y Blepharocalyx salicifolius y Myrcianthes pseudomato son árboles de gran porte, de 15 a $30 \mathrm{~m}$ y 8 a $12 \mathrm{~m}$ de altura respectivamente, con presencia de inflorescencias en el período de octubre a diciembre (Digilio \& Legname, 1966).
En relación al resto de las especies arbóreas con clases de frecuencias de menor importancia o secundario, Handroanthus ochraceus y Sambucus peruviana es la primera vez que son citadas en estudios en esta índole. Handroanthus ochraceus florece entre agosto y octubre y es considerada una especie que aporta néctar a la colmena; Sambucus peruviana, dada su floración de noviembre a enero, constituye un importante recurso durante la época estival.

Lo observado en relación a Ilex argentina, especie que florece de octubre a diciembre y cuya presencia en las muestras se caracterizó como un recurso muy frecuente, se destaca su importancia nectarífera para las colmenas, a diferencia de lo encontrado en las mieles de Apis mellifera (Sánchez, 2013), donde fue hallada con una presencia traza $(<3 \%)$ y poco frecuente. En el caso de Parapiptadenia excelsa, especie que florece entre setiembre y noviembre y que aporta néctar a la colmena, también ha sido observada como un tipo polínico significativo en mieles y cargas corbiculares de A. mellifera en ambientes de Chaco (Burgos \& Sánchez, 2014; Burgos et al., 2015) y de Yungas más australes (Sánchez, 2013; Méndez, 2014), como también en mieles de Tetragonisca angustula (Flores \& Sánchez, 2010).
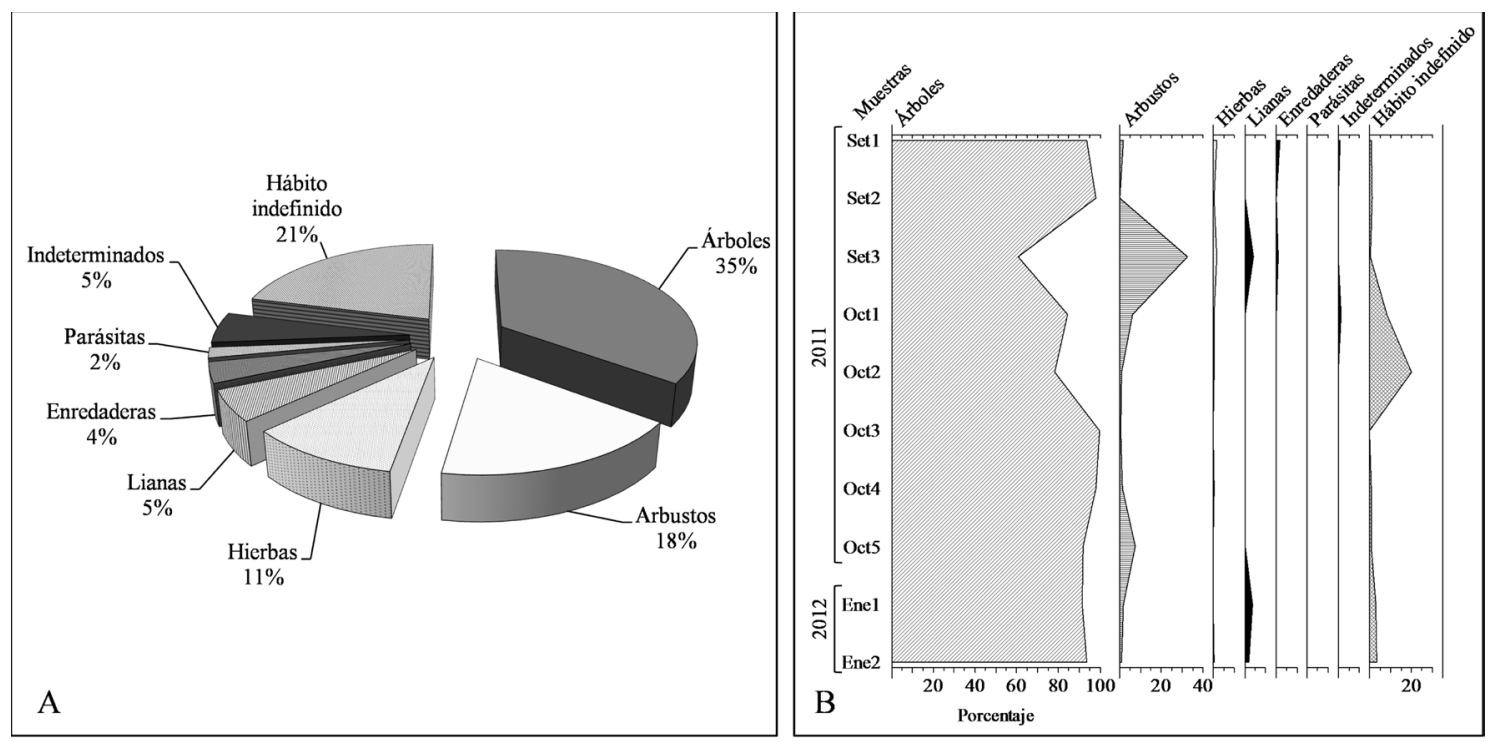

Fig. 9. Representatividad de los tipos polínicos en las mieles de Plebeia intermedia, de acuerdo al hábito de la especie vegetal, según su frecuencia porcentual (A) y abundancia porcentual (B). 
Entre las plantas arbustivas se destacaron los tipos polínicos Eupatorium y Senecio, mientras que en el caso de los tipos polínicos identificados a nivel de familia botánica, fueron relevantes los pólenes de Boraginaceae y Rhamnaceae. En concordancia, en otras regiones y otros biomas, existen antecedentes de uso por parte de Apis mellifera; por ejemplo, en estudios realizados en ambientes yungueños (Sánchez, 2013; Méndez, 2014) y chaqueños (Burgos \& Sánchez, 2014; Salgado et al., 2014), del territorio argentino, se destaca la importancia de integrantes de estos géneros y familias botánicas como recursos nectaríferos, presentes en las mieles como pólenes trazas a dominantes. Respecto a tipos polínicos procedentes de especies de lianas, Paullinia se destacó con clases de frecuencias de menor importancia, citándose por primera vez para estudios de esta índole. Sobre la base de estos antecedentes, se plantea la necesidad de estudios complementarios que revelen la importancia de sus especies para las colmenas de Plebeia intermedia.

Por otra parte y como se mencionó anteriormente, los tipos polínicos de plantas exóticas y/o cultivadas solo representan el 2\% del total observado (Fig. 6) y siempre se encontraron presentes como elementos trazas $(<3 \%)$ en las muestras. En esta categoría se encuentra el tipo polínico Citrus; a pesar de su baja representatividad en las muestras, se considera un recurso importante de néctar para las abejas melíferas en general. En ese contexto, por ejemplo Chacoff (2006), al estudiar los polinizadores de cultivos de $C$. paradisi Macf. (Rutaceae), registró la visita de diversas abejas utilizándola como fuente de néctar; asimismo en estudios realizados en mieles de Apis mellifera en ambientes del Chaco (Burgos \& Sánchez, 2014) y de Tetragonisca angustula en sectores de Yungas (Flores \& Sánchez, 2010), este género botánico resultó ser un recurso nectarífero importante, clasificado desde polen traza a dominante. En el área estudiada, no existen plantaciones de cítricos, pero entre la matriz boscosa se encuentran esporádicamente individuos asilvestrados de Citrus aurantifolia (Christm.) Swingle (Rutaceae) y de C. sinensis (L.) Osbeck (Rutaceae), ambas especies cultivadas en dos localidades cercanas - El Lipeo y Cidras ubicadas en un nivel altitudinal ligeramente menor (Hilgert, 1999), que contribuyen eventualmente en la elaboración de las mieles.

Los tipos polínicos de plantas con polinización anemófila o sin néctar (Alnus acuminata, Celtis, Juglans australis, tipo Rumex y Zea mays), son elementos habitualmente observados en las mieles de A. mellifera (Sánchez, 2013; Méndez, 2014) y de T. angustula (Flores \& Sánchez, 2010); si bien, fueron excluidos de la caracterización botánica de las muestras, las posibles vías de ingreso en las mieles de Plebeia intermedia pueden justificarse a través de dos potenciales explicaciones:

1 - un ingreso ocurrido a través del aire: al ser considerados elementos aeropolínicos frecuentes durante los meses que florecen (García, 2006; Torres \& Flores, 2013; Torres et al., 2014), su arribo posiblemente coincidió con los momentos de meleo efectuados por los pobladores.

2-un ingreso intranidal: debido a la importancia que adquieren las plantas como recursos poliníferos para las abejas, la presencia de los pólenes en las mieles estaría respaldada por una contaminación ocurrida en el interior de las colmenas, desde los sitios de acopio del polen corbicular, o mediante el reciclaje de los potes de almacenamiento con residuos basales de polen, como ocurrió en colmenas de Scaptotrigona jujuyensis del Chaco argentino (Basilio et al., 2013).

Los tipos polínicos Alnus acuminata, Celtis, Juglans australis y tipo Rumex, corresponden a especies presentes en los distritos Selva Montana y Bosque Montano de las Yungas (Brown, 2007; Brown \& Malizia, 2007) y se conocen antecedentes de uso polinífero por colmenas de Apis mellifera (Méndez, 2014) y Plebeia intermedia (Flores, inédito). En el caso de Zea mays, la especie es uno de los cultivos familiares en la región (Hilgert, 1999; Hilgert et al., 2013). Al carecer de nectarios y el hecho de constituir un recurso primordialmente polinífero, su presencia en la miel probablemente también se deba a una contaminación en el interior de las colmenas. En estudios realizados en colmenas de $A$. mellifera, ubicadas en ambientes chaqueños, se observa la presencia de esta especie cultivada en las muestras de polen corbicular analizadas, en ocasiones como tipo polínico dominante o secundario (Burgos et al., 2015), así como también su presencia en las muestras de miel (Burgos \& Sánchez, 2014). Posiblemente la especie de melipona que nos ocupa, utilice este recurso de manera análoga, lo cual debe comprobarse con el examen de las cargas corbiculares en futuros trabajos. 
Este trabajo constituye un primer aporte al conocimiento sobre las mieles de Plebeia intermedia en el período primavera - verano. Los datos preliminares obtenidos en este estudio, destacan una cierta especialización de $P$. intermedia por determinados recursos tróficos de plantas arbóreas, lo cual disiente del hábito de forrajeo generalista asignado a las especies de meliponas (Heithaus, 1979; Ramalho et al., 1990). Además, una especialización orientada al forrajeo de recursos dispuestos en floraciones en masa, coincidente a los estudios de visitas florales de especies de meliponas de los bosques de Brasil, donde exhiben dominancia del estrato superior de árboles sobre otros insectos melíferos (Ramalho, 2004), como también en meliponas de la región Chaqueña argentina, donde se observó una alta frecuencia de visitas hacia plantas de hábito herbáceo (Vossler, 2012). No obstante, este comportamiento de forrajeo probablemente represente una especialización de los individuos de las colonias en cortos períodos de tiempo (Slaa, 2003), lo cual debe corroborarse con el análisis de mieles de otras épocas del año y el examen de las cargas corbiculares. En efecto, estos nuevos estudios propuestos a futuro, complementados a los resultados alcanzados en este artículo, permitirán comprender concretamente si el comportamiento de Plebeia intermedia consiste en una apropiación de los nichos tróficos de otros visitantes generalistas, o a la existencia de una coevolución difusa con las plantas con floraciones en masa presentes en ambientes de Yungas.

\section{Agradecimientos}

Agradecemos al CONICET por el apoyo otorgado para la realización de este trabajo, a la Administración de Parques Nacionales y sus integrantes que facilitaron la realización del mismo. Al Lic. Leopoldo J. Álvarez, de la División de Entomología del Museo de La Plata - Universidad Nacional de La Plata, por la identificación taxonómica de la especie de melipona, y a los pobladores de la localidad de Baritú por su buena predisposición en las entrevistas y colección de las muestras de miel. Un especial agradecimiento a los revisores, con sus comentarios este trabajo mejoró sustancialmente la presentación de sus resultados.

\section{Bibliografía}

AGUILERA PERALTA, F. J. \& U. FERRUFINO. 2004. Cómo criar abejas sin aguijón. Ed. ASEO, Santa Cruz de la Sierra, Bolivia.

BASILIO, A. M., C. SPAGARINO, L. LANDI \& B. ACHÁVAL. 2013. Miel de Scaptotrigona jujuyensis en dos localidades de Formosa, Argentina. En: VIT, P. \& D.W. ROUBIK (eds.), Stingless bees process honey and pollen in cerumen pots, pp 1-8. Facultad de Farmacia y Bioanálisis, Universidad de Los Andes. Mérida, Venezuela. http://www.saber.ula.ve/ handle/123456789/3529.

BROWN, A. D. 2007. Caracterización ambiental ecoregional. En: BROWN, A. D., M. GARCÍA MORITÁN, B. N. VENTURA, N. I. HILGERT \& L. R. MALIZIA (eds.), Finca San Andrés. Un espacio de cambios ambientales y sociales en el Alto Bermejo, pp 25-66. Ediciones del Subtrópico, Tucumán.

BROWN, A. D. \& L. MALIZIA. 2007. Finca San Andrés: realidad ambiental de una propiedad - cuenca en el Noroeste Argentino. En: BROWN, A. D., M. GARCÍA MORITÁN, B. N. VENTURA, N. I. HILGERT \& L. R. MALIZIA (eds.), Finca San Andrés. Un espacio de cambios ambientales y sociales en el Alto Bermejo, pp 85-100. Ediciones del Subtrópico, Tucumán.

BURGOS, M. G. \& A. C. SÁNCHEZ. 2014. Preferencias alimenticias en las mieles inmaduras de Apis mellifera en el Chaco Serrano (Jujuy, Argentina). Bol. Soc. Argent. Bot. 49: 41-50.

BURGOS, M. G., A. C. SÁNCHEZ \& L. C. LUPO. 2015. Análisis polínico de cargas corbiculares de Apis mellifera del Chaco Serrano, Jujuy (Argentina). Lilloa 52: 3-11.

CABRERA, A. L. 1971. Fitogeografía de la República Argentina. Bol. Soc. Argent. Bot. 14: 1 - 42.

CAMARGO, J. M. F. \& S. R. M. PEDRO. 2013. Meliponini Lepeletier, 1836. En: MOURE, J. S., D. URBAN \& G. A. R. MELO (Orgs), Catalogue of Bees (Hymenoptera, Apoidea) in the Neotropical Region. http://www. moure.cria.org.br/catalogue. (Cons. 10/06/2015).

CHACOFF, N. P. 2006. Los Ecosistemas Naturales como fuente de Polinizadores para Citrus paradisi en el Pedemonte de las Yungas. Tesis doctoral, Universidad Nacional de Tucumán.

COLLESELLI, L. M. D. \& N. N. GÓMEZ DE OLIVERA. 2008. Las yateí (Tetragonisca angustula) en el Refugio Privado de Vida Silvestre Yacutinga, Misiones, Argentina. Tesis Técnico Universitario Guardaparques. Universidad Nacional de Misiones.

CONCEIÇÃO, P. J. 2013. Levantamento florístico $e$ perfil botânico do pólen (samburá) da abelha Melipona quadrifasciata anthidioides Lepeletier, 1836 (Hymenoptera: Apidae) da Região Semiárida, Estado da Bahia. Tesis do Mestrado em Ciências Agrárias, 
Universidade Federal do Recôncavo da Bahia.

CORTOPASSI LAURINO, M. \& M. RAMALHO. 1988. Pollen harvest by Africanized Apis mellifera and Trigona spinipes in Sao Paulo: botanical and ecological views. Apidologie 19: 1-24.

DA SILVA, C. I. 2014. Catálogo polínico das plantas usadas por abelhas no campus da USP de Ribeirão Preto. Edittorial Holos, São Paulo. Brasil.

DE KLERK, P. \& H. JOOSTEN. 2007. The difference between pollen types and plant taxa: a plea for clarity and scientific freedom. Eiszeitalter und Gegenwart/ Quaternary Science Journal 56: 162-171.

DIGILIO, A. \& P. LEGNANE. 1966. Los árboles indígenas de Tucumán. Opera Lilloana 15: 1-283.

ERDTMAN, G. 1960. The acetolysis method, a revised description. Svensk. Bot. Tidskr. 54: 561-564.

FELLER DEMALSY, M., J. PARENT \& A. STRACHAN. 1987. Microscopic analysis of honeys from Alberta, Canada. J. Apic. Res. 26: 123-132.

FLORES, F. F. \& A. C. SÁNCHEZ. 2010. Primeros resultados de caracterización botánica de mieles de Tetragonisca angustula Latreille (Apidae, Meliponinae) criadas en la localidad Los Naranjos - Orán - Salta. Bol. Soc. Argent. Bot. 45: 81-91.

GARCÍA, M. E. 2006. Lluvia polínica en selvas montanas de la provincia de Tucumán, Argentina. Rev. Mus. Argentino Cs. Nat. 8: 159-164.

GRIMM, E. 2011. Tilia Software. Versión 1.7.16. Illinois State Museum. Research \& Collection Center. Springfield, Illinois.

HEITHAUS, E. R. 1979. Flower-feeding specialization in wild bee and wasp communities in seasonal neotropical habitats, Oecologia 42: 179-194.

HEUSSER, C. J. 1971. Pollen and spores of Chile. Tucson, Arizona.

HILGERT, N. I. 1999. Las plantas comestibles en un sector de las Yungas meridionales (Argentina). Anales Jard. Bot. Madrid 57: 117-138.

HILGERT, N. I., F. ZAMUDIO, V. FURLAN \& L. CARIOLA. 2013. The key role of cultural preservation in maize diversity conservation in the Argentine Yungas. Evidence-Based Complementary and Alternative Medicine. DOI: 10.1155/2013/7327602013.

HILGERT MOREIRA, S. B., C. A. NASCHER, S. M. CALLEGARI JACQUES \& B. BLOCHTEIN. 2013. Pollen resources and trophic niche breadth of Apis mellifera and Melipona obscurior (Hymenoptera, Apidae) in a subtropical climate in the Atlantic rain forest of southern Brazil. Apidologie. DOI: 10.1007/ s13592-013-0234-5.

IWAMA, S \& T. S. MELHEM. 1979. The Pollen Spectrum of the honey of Tetragonisca angustula angustula Latreille (Apidae, Meliponinae). Apidologie 10: 275295.
JOOSTEN, H. \& P. DE KLERK. 2002. What's in a name? Some thoughts on pollen classification, identification, and nomenclature in Quaternary palynology. Review of Palaeobotany and Palynology 122: 29-45. DOI:10.1016/S0034-6667(02)00090-8.

KNOLL, F. D. R. N., L. R. BEGO \& V. L. IMPERATRIZ FONSECA. 1994. As abelhas em áreas urbanas. Um estudo no Campus da Universidade de São Paulo. En: PIRANI, J. R. \& M. CORTOPASSI LAURINO (eds.), Flores e abelhas em São Paulo, pp 31-42. Edusp, São Paulo, Brasil.

LOUVEAUX, J., A. MAURIZIO \& G. VORWHOL. 1978. Methods of Melissopalynology by International Commission of Bee Botany of IUBS. Bee World 59: 139-157.

MAIA SILVA, C., C. I. DA SILVA, M. HRNCIR, R. T. QUEIROZ \& V. L. IMPERATRIZ FONSECA. 2012. Guia de plantas visitadas por abelhas na Caatinga. 1ra. ed. Editora Fundação Brasil Cidadão, Fortaleza.

MARKGRAF, V. \& H. D’ANTONI. 1978. Pollen Flora of Argentina. The University of Arizona Press. Arizona.

MARTÍNEZ HERNÁNDEZ, E., J. I. CUADRIELLO AGUILAR, O. TÉLLEZ VALDEZ, E. RAMÍREZ ARRIAGA, M. S. SOSA NÁJERA, J. E. M. MELCHOR SÁNCHEZ, M. MEDINA CAMACHO \& M. S. LOZANO GARCÍA. 1993. Atlas de las plantas y el polen utilizados por las cinco especies principales de abejas productoras de miel en la región del Tacaná, Chiapas, México. Ed. Instituto de Geología, UNAM, México.

MÉNDEZ, M. V. 2014. Recursos nectariferos y poliníferos utilizados por Apis mellifera L. en la localidad de Tilquiza, Jujuy, Argentina. Tesina de grado, Universidad Nacional de Jujuy.

MENEZES PEDRO, S. R. de \& J. M. F. de CAMARGO. 1991. Interactions on floral resources between the Africanized honey bee Apis mellifera L. and the native bee community (Hymenoptera: Apoidea) in a natural "cerrado" ecosystem in southeast Brazil. Apidologie 22: 397-415.

MICHENER, C. D. 2007. The bees of the world. $2^{\text {nd }}$ ed. The Johns Hopkins University Press, Baltimore.

MONTEIRO, D. \& M. RAMALHO. 2010. Abelhas generalistas (Meliponina) e o sucesso reprodutivo de Stryphnodendron pulcherrimum (Fabales: Mimosaceae) como florada em massa na Mata Atlantica, BA. Neotropical Entomology 39: 519-526.

MORALES, J. M. \& A. D. BROWN. 1998. Bosques montanos con diferente intensidad de explotación. Bosques 17: 51-52.

MORALES, J. M., M. SIROMBRA \& A. D. BROWN. 1995. Riqueza de árboles de Yungas argentinas. En: BROWN, A. D. \& H. R. GRAU (eds.), Investigación, Conservación y Desarrollo en las Selvas Subtropicales de montaña, pp. 163-170. Laboratorio 
de Investigaciones Ecológicas de las Yungas, UNT, Tucumán.

PINAZO, M. A., N. I. GASPARRI, J. F. GOYA \& M. F. ARTURI. 2003. Caracterización estructural de un bosque de Podocarpus parlatorei y Juglans australis en Salta, Argentina. Rev. biol. trop. 51: 361-368.

PIRE, S. M., L. M. ANZÓTEGUI \& G. A. CUADRADO. 1998. Flora Polínica del Nordeste Argentino 1. EUDENE-UNNE, Corrientes.

PIRE, S. M., L. M. ANZÓTEGUI \& G. A. CUADRADO. 2001. Flora Polínica del Nordeste Argentino 2. EUDENE-UNNE, Corrientes.

PIRE, S. M., L. M. ANZÓTEGUI \& G. A. CUADRADO. 2006. Flora Polínica del Nordeste Argentino 3. EUDENE-UNNE, Corrientes.

RAMADORI, D. 1995. Agricultura migratoria en el Valle del Río Baritú, Santa Victoria, (Salta). En: BROWN, A. D. \& H. R. GRAU (eds.), Investigación, Conservación y Desarrollo en las Selvas Subtropicales de montaña, pp. 205-214. Laboratorio de Investigaciones Ecológicas de las Yungas, UNT, Tucumán.

RAMALHO, M. 2004. Stingless bees and mass flowering trees in the canopy of Altlantic Forests: a tight relationship. Acta bot. bras. 18: 37-47.

RAMALHO, M., V. L. IMPERATRIZ FONSECA, A. KLEINEKT GIOVANNINI \& M. CORTOPASSI LAURINO. 1985. Exploitation of floral resources by Plebeia remota Holmberg (Apidae, Meliponinae). Apidologie 16: 307-330.

RAMALHO, M., KLEINERT GIOVANNINI, A. \& V. L. IMPERATRIZ FONSECA. 1990. Important bee plants for stingless bees (Melipona and Trigonini) and Africanized honeybees (Apis mellifera) in neotropical habitats: a review. Apidologie 21: 469-488.

ROIG ALSINA, A., F. G. VOSSLER \& P. G. GENNARI. 2013. Stingless bees in Argentina. En: VIT, P., PEDRO, S. R. M. \& D. W. ROUBIK (eds.), Pot Honey. A legacy of stingless bees, pp. 125-134. Springer Verlag, New York.

SAlGADO, C. R., G. PIESZKO \& M. C. TELLERÍA. 2014. Aporte de la Melisopalinología al conocimiento de la flora melífera de un sector de la Provincia Fitogeográfica Chaqueña, Argentina. Bol. Soc. Argent. Bot. 49: 513-524.

SÁNCHEZ, A. C. 2013. Caracterización botánica y geográfica de las mieles de Apis mellifera L. en la provincia de Jujuy. Tesis doctoral, Universidad Nacional de Salta.
SLAA, E. J. 2003. Foraging ecology of stingless bees: from individual behavior to community ecology. Doctoral dissertation, Utrecht University, The Netherlands.

SPARAGNINO, C., P. CHIANETTA \& A. M. BASILIO. 2014. Especies arbóreas utilizadas por abejas meliponas (Apidae: Meliponini) en el bosque chaqueño semiárido en Formosa (Argentina). Agronomía \& Ambiente 34: 85-91.

TORRES, G. R. \& F. F. FLORES. 2013. Reporte de polen atmosférico durante la estación primaveral de 2007 en la ciudad de San Salvador de Jujuy, Argentina. Rev. Mus. Argentino Cs. Nat. 15: 175-182.

TORRES, G. R., C. F. PÉREZ, L. C. LUPO \& O. G. MARTÍNEZ. 2014. Registro preliminar del depósito atmosférico anual de esporas de helechos de las Yungas sobre un gradiente ambiental en la cuenca media del Río Perico (Jujuy, Argentina). Bol. Soc. Argent. Bot. 49: 503-512.

VOSSLER, F. G. 2012. Flower visits, nesting and nest defence behavior of stingless bees (Apidae: Meliponini): suitability of the bee species for meliponiculture in the Argentinean Chaco region. Apidologie 43:139-161.

VOSSLER, F. G. 2015. Small pollen grain volumes and sizes dominate the diet composition of three South American subtropical stingless bees. Grana 54: 68-81.

VOSSLER, F. G., M. C. TELLERÍA\& M. CUNNINGHAM. 2010. Floral resources foraged by Geotrigona argentina (Apidae, Meliponini) in the Argentine Dry Chaco forest. Grana 49: 142-153.

VOSSLER, F. G., M. C. TELLERÍA\& M. CUNNINGHAM. 2014. Variability of food stores of Tetragonisca fiebrigi (Schwarz) (Hymenoptera: Apidae: Meliponini) from the Argentine Chaco based on pollen analysis. Sociobiology 61: 449-460. DOI: 10.13102/ sociobiology.v61i4.449-460.

ZULOAGA, F. O., O. N. MORRONE, M. J. BELGRANO, C. MARTICORENA \& E. MARCHESI. (eds). 2008. Catálogo de las Plantas Vasculares del Cono Sur (Argentina, Sur de Brasil, Chile, Paraguay y Uruguay). Monogr. Syst. Bot. Missouri Bot. Gard. 107: I-XCVI, 1-983; 107: I-XX, 985-2286; 107: I-XXI, 2287-3348. http://www2.darwin.edu.ar/Proyectos/FloraArgentina/ FA.asp (Cons. 23/07/2015).

Recibido el 22 de abril de 2015, aceptado el 03 de setiembre de 2015 . 
